Conclusion The development of CIA and autoimmune responses in MRL/lpr mice were markedly suppressed by SX-5452 via inhibition of anti-CII/anti-DNA antibodies formation. Since SX5452 exerts no overt toxicity after repeated administration in mice, it is an interesting candidate for therapeutic use in rheumatoid arthritis.

\section{THU0089 INHIBITION OF RHEUMATOID ARTHRIRTIS SYNOVIOCYTES GROWTH BY TAURINE CHLORAMINE}

${ }^{1} \mathrm{E}$ Kontny, ${ }^{2} \mathrm{~J}$ Kowalczewski, II Janicka, ${ }^{1} \mathrm{M}$ Kurowska, ${ }^{3} \mathrm{~J}$ Marcinkiewicz, ${ }^{1} \mathrm{~W}$ Maslinski. ${ }^{1}$ Department of Pathophysiology and Immunology; ${ }^{2}$ Clinic of Orthopaedy, Institute of Rheumatology, Warsaw; ${ }^{3}$ Department of Immunology, Jagiellonian University Medical College, Cracow, Poland

\subsection{6/annrheumdis-2001.966}

Background Hyperplasia of synovial membrane, a characteristic feature of rheumatoid arthritis (RA), is proposed to result from either the enhanced proliferation of fibroblast-like synoviocytes (FLS) or the resistance of these cells to apoptotic death. Therapeutic approaches to inhibit an excessive growth of RA FLS are not satisfactory. We have previously shown that taurine chloramine (Tau-Cl), a chlorinated derivative of a dominant free amino acid taurine (Tau), formed by neutrophils during respiratory burst, inhibits FGF-triggered proliferation of RA FLS (Arthritis Rheum 1999;42:2552).

Objectives The aim of present study was to investigate the mechanism of Tau-Cl inhibition of RA FLS growth.

Methods FLS, isolated from the synovial membrane of RA patients, were stimulated in vitro for $18-72 \mathrm{~h}$ with $10 \mathrm{ng} / \mathrm{ml}$ of either rhPDGF or rhTNF- $\alpha$. Tau or Tau-Cl were added (at 100$500 \mu \mathrm{M}$ concentration) together with the stimuli. Cell proliferation was determined by incorporation of $3 \mathrm{H}-\mathrm{TdR}$. To evaluate the cytotoxicity of tested compounds, and to estimate the recovery of the cells from the cultures, the lactate dehydrogenase $(\mathrm{LDH})$ activity was measured in culture supernatants and in cell lysates, respectively. The expression of proteins regulating the cell-cycle progression or apoptosis, was estimated by Western bloting.

Results The rate of spontaneous proliferation of FLS ranged from 176 to $4639 \mathrm{cpm}$. Both, PDGF and TNF- $\alpha$ raised it significantly by 6 and 2 times, respectively. Tau was neither cytotoxic nor affected the recovery/proliferation of RA FLS. In contrast, Tau-Cl inhibited both the proliferation and the cell recovery in a dose-dependent manner. Independent of the type of stimulus, both responses were reduced by $70 \%$ in the presence of 250 $\mu \mathrm{M}$, and were almost completely blocked (by 90\%) in the presence of $500 \mu \mathrm{M}$ concentration of Tau-Cl. At the same concentrations $(250$ and $500 \mu \mathrm{M})$ Tau-Cl inhibited PDGF- and TNF- $\alpha$ triggered expression of PCNA (proliferating cell nuclear antigen) but has no effect on the expression of either the Cdk-4 (the cyclin-dependent kinase-4), Cdk-inhibitors (p16, p21, p27) or the anti-apoptotic, Bcl-2, protein. At the late time points (48-72 hrs) only the higher $(500 \mu \mathrm{M})$ concentration of Tau-Cl exerted cytotoxic (50\%) effect.

Conclusion We report that $\mathrm{Tau}-\mathrm{Cl}$, a physiologic factor originated from activated neutrophils, blocks proliferation of RA FLS by affecting the common event critical for the cell cycle progression: the expression of PCNA protein, known to act as a cofactor for DNA polimerase $\delta$. Thus, Tau-Cl might be promising therapeutic agent for RA. Whether Tau-Cl triggers apoptosis of RA FLS is under investigation.

\section{THU0090 TAP1 AND TAP2 GENE POLYMORPHISM IN RHEUMATOID ARTHRITIS IN EASTERN FRENCH POPULATION}

${ }^{1} S$ Zhang, ${ }^{2}$ C Chabod, ${ }^{3} \mathrm{~A}$ Penfornis, ${ }^{2} \mathrm{P}$ Tiberghien, ${ }^{1} \mathrm{D}$ Wendling, ${ }^{1} \mathrm{E}$ Toussirot. ${ }^{1}$ Rheumatology; ${ }^{2}$ Immunogenetics; ${ }^{3}$ Endocrinology, University Hospital Minjoz, Besançon, France

10.1136/annrheumdis-2001.967

Background The Transporter associated with antigen processing (TAP) gene products are involved in the processing of endogenous peptides that bind to human leukocyte antigen (HLA) class I molecules and polymorphism within these genes could alter the immune responses, the phenomenon relevant to the development of autoimmunes diseases.

Objectives In this study, we examined the polymorphism of TAP1 and TAP2 genes in patients with rheumatoid arthritis (RA).

Methods TAP1 and TAP2 typing was performed in 138 white RA patients and 100 healthy controls, all of them originating from Eastern France. TAP1 polymorphic residues at position 333 and 637 and amino acid variants 379, 565, 651 and 665 in TAP2 gene were determined using amplification refractory mutation system (ARMS)- PCR. This method enabled us to determine 4 TAP1 alleles (TAP1A to TAP1D) and 8 TAP2 alleles (TAP2A to TAP2H). All patients and controls had been HLA DRB1* genotyped.

Results The polymorphic residues TAP1/333 and TAP1/637 did not show any difference in their distribution between patients and controls.

Similar findings were observed for TAP2/379 and TAP2/665. However, we found an increased frequency of Thr homozygosity and heterozygosity at position 565 in TAP2 gene (RA vs Controls: $25.3 \%$ vs $14 \%$; p: 0.032;OR: 2.09;CI: 1.01-4.38). Similarly, the prevalence of subjects who were homozygote and heterozygote for Cys/651 was increased in the RA group (RA vs Controls: $22.4 \%$ vs $11 \%$; p:0.02). The frequency of TAP2F was higher in RA patients $(24.5 \%)$ as compared with controls (11.3\%), without significance after correction ( $\mathrm{p}: 0.029$; $\mathrm{p}$ corr: $0.17)$.

Finally, we found no linkage disequilibrium between DRB1* associated -alleles and amino acid substitution $\mathrm{Thr} / 665$ whereas Cys/651 was not independent of DRB1*04, a strongly RA-linked allele.

Only a few patients expressing Thr at position 565 in TAP2 gene were associated with severe clinical manifestations.

Conclusion We evidenced in this study an association between a particular amino acid residue, namely Thr/665 in TAP2 gene, and RA.

This association was found to be weak and did not seem to be a predictor for the severity of the disease.

\section{THU0091 IL-8 PRODUCTION INDUCED BY PROTEIN I/II, A MODULIN FROM ORAL STREPTOCOCCI, IN SYNOVIOCYTES FROM RHEUMATOID ARTHRITIS (RA) PATIENTS INVOLVES THE MAPKS AND FAK SIGNALLING PATHWAYS AND NF-KB}

${ }^{1} \mathrm{~L}$ Neff, ${ }^{1} \mathrm{M}$ Zeisel, ${ }^{2} \mathrm{FX}$ Limbach, ${ }^{1} \mathrm{JP}$ Klein, 'D Wachsmann, ${ }^{2} \mathrm{~J}$ Sibilia. ${ }^{1}$ Pharmacology; ${ }^{2}$ Rheumatology, CHU Hautepierre, Strasbourg, France

10.1136/annrheumdis-2001.968 
Background Protein I/II, an adhesin from viridans streptococci, commensal bacteria of the oral cavity, can stimulate different cells including endothelial cells. After binding to its receptor, integrin A5B1, protein $\mathrm{I} / \mathrm{II}$ promotes release of IL-6 and IL-8 from endothelial cells, which contributes to the recruitment of circulating leukocytes from the blood into tissues. IL-8 has recently by associated with the pathogenesis of various chronic inflammatory disorders such as RA. IL- 8 could be produced in response to commensal bacteria which could disseminate through bacteremia.

Objectives To study the interactions of protein I/II with fibroblast-like synoviocytes from RA patients.

Methods

Results

- Protein I/II increased the IL-8 synthesis of fibroblast-like synoviocytes through transcriptional up-regulation of the IL-8 gene (ELISA and RT-PCR).

- IL-8 synthesis was inhibited by the tyrosine kinase inhibitor herbimycin A and by inhibitors of MAPKs such as apigenin and PD 98059, while focal adhesion kinase (FAK) and ERK1/2 phosphorylation was increased by Protein I/II.

- Protein I/II increased activated nuclear translocation of the transcription factor NF-KB (EMSA with an NF-KB specific probe). This was confirmed by inhibition with curcumin, an inhibitor of NF-KB, which blocked IL-8 release from protein I/II-activated synoviocytes.

\section{Conclusion}

- After binding to synoviocytes, protein I/II induces IL-8 release by activaing the FAK and MAPKs signalling pathways.

- The transcription factor NF-KB plays a major role in the upregulation of IL-8 synthesis and release mediated by NF-KB.

- Synoviocytes activation could be triggered by commensal bacteria, which could spread to the joints through bacteremia in RA and others forms of arthritis.

\section{THU0092 THE L1 MRNA IN RHEUMATOID ARTHRITIS ENCODES A FUNCTIONAL RETROTRANSPOSABLE ELEMENT}

${ }^{1} \mathrm{M}$ Neidhart, ${ }^{1} \mathrm{~J}$ Rethage, ${ }^{1} \mathrm{O}$ Distler, ${ }^{2} \mathrm{MT}$ Peyer, ${ }^{3} \mathrm{ME}$ Billingham, ${ }^{1} \mathrm{BA}$ Michel, ${ }^{1}$ RE Gay, ${ }^{1} \mathrm{~S}$ Gay. ${ }^{1}$ Rheumatology, University Hospital, Zurich, Switzerland; ${ }^{2}$ Institute for Plant Physiology, University of Montreal, Montreal, Canada; ${ }^{3}$ Veterinary School, University of Bristol, Bristol, UK

\subsection{6/annrheumdis-2001.969}

Background L1 retrotransposons have been detected in rheumatoid arthritis (RA) at sites of joint destruction.

Objectives To caracterise the L1 mRNA in RA.

Methods RNA was isolated from the synovial tissue of a 35years old woman with RA. The complete L1 sequence was determined by 3 ?/5?-RACE and compared with functional L1 retrotransposons in plants, animals and humans using 50\% consensus trees of the elements and the neighbour-joining method. Methylation of the genomic 5 ?UTR was determined by the sodiumdisulfite/PCR method. In situ hybridization was performed on synovial tissues with riboprobes recognising the ORF1 of this element to detect intact L1 elements.

Results A complete L1 mRNA was obtained with the 5?UTR (including a hypoxia inducing factor-responding element and numerous CpG islands), an ORF1 (encoding the p40/RNA binding protein) and an ORF2 (encoding the p150/endonuclease and reverse transcriptase protein). In RA, the genomic 5?UTR appeared hypomethylated, compared to normal control DNA. In situ hybridization showed that the L1 ORF1 transcript is expressed in the sub-lining of patients with RA. Phylogenic trees showed that the 5?UTR nucleotide sequence and the ORF1 peptide sequence have high similarities with human L1.39 and L1.25, two functional L1 retrotransposons, and the ORF2 peptide sequence resembled the M22334 L1 insertion in type A haemophilia. The endonuclease region of ORF2 appears to be highly conserved in functional L1s, but as shown by a phylogenic tree, the small difference appears to be a specific marker for evolution, discriminating L1s in plants, rodents, primates and humans.

Conclusion Intact L1 elements are expressed in the synovial tissue of patients with RA most likely following hypomethylation of their 5?UTR. The L1 mRNA transcript belongs to the functional L1 retrotransposons, including elements producing insertional mutations. Thus, functional L1s could be involved in the transformed phenotype of some synovial cells.

\section{REFERENCE}

1 Neidhart M, et al. Arthritis Rheum. 2000;43:2634-47

\section{THU0093 RHEUMATOID SYNOVIA AND ACANTHAMOEBA POLYPHAGA: IMMUNOHISTOCHEMICAL EVIDENCE OF CROSSREACTIVITY}

${ }^{1} \mathrm{~S}$ Jeansson, ${ }^{2} \mathrm{~T}$ Klingen, ${ }^{2} \mathrm{~B}$ Roald, ${ }^{3} \mathrm{E}$ Rud, ${ }^{3} \mathrm{~T}$ Kvien. ${ }^{1}$ Department of Microbiology; ${ }^{2}$ Department of Pathology, Ullevaal University Hospital; ${ }^{3}$ Department of Rheumatology, Diakonhjemmet Hospital, Oslo, Norway

10.1136/annrheumdis-2001.970

\section{Background}

Objectives The initiation event in rheumatoid arthritis (RA) may be triggered by an infection and an association to various microorganisms has been discussed. Micro-organisms capable of persistence, such as parvovirus B19 and Epstein-Barr virus, are of special interest. Features which might be expected of any microorganism associated with RA, such as persistence, are also found in the protozoan Acanthamoeba polyphaga (AP). The aim of the present study was by means of immunohistochemical techniques, to search for epitopes in synovial tissue from RA patients and non-RA patients reacting/crossreacting with an AP specific monoclonal antibody.

Methods Patient material:Synovia and pannus was removed from the knee during arthroscopy or open joint surgery on 57 patients in a single surgical unit at Diakonhjemmet Hospital in Oslo. These patients were classified into three groups. One had a clinical diagnosis of RA $(n=30)$ according to the criteria of the American College of Rheumatology. The other 27 patients had degenerative joint disease $(n=17)$, or other inflammatory arthropathies $(\mathrm{n}=10)$. The specimens were evaluated by light microscopy and immunohistochemistry. Monoclonal antibodies (MAb) against AP were produced by standard methods. A MAb reactive with the cytoplasm of AP and non-reactive with normal human skin biopsies was selected for use in immunohistochemistry.

Results Synovial membranes from RA patients demonstrated a distinct immunoreactivity whereas the reactivity in non-RA patients was weak. RA patients often demonstrated reactivity in the nuclear membrane in addition to cytoplasm and nucleus. The immunereactivity in non-RA patients was restricted to cytoplasm and nucleus in endothelial cells, pericytes and fibroblasts. Conclusion Our findings indicates the presence of an epitope crossreactive between synovia and the protozoan AP. Epitopes 